\title{
Corrigendum
}

\section{Validation of a semi-quantitative food-frequency questionnaire used among 2-year-old Norwegian children - Corrigendum}

\author{
LF Andersen, B Lande, K Trygg and G Hay
}

doi:10.1079/PHN2004613, Public Health Nutrition 2004; 7(6): 757-64. Published online by Cambridge University Press 2 Jan 2007

There is a programming error which resulted in a systematic error in the data previously published from the validation study of a semi-food frequency questionnaire (SFFQ) used among Norwegian 2-year-olds. Specifically, the error caused the food intake estimates for the girls (from the SFFQ) to be divided with 2 . Thus, this error has consequences for the absolute intake values for the SFFQ presented in original Table 2 (nutrient intake) and in original Table 5 (food intake). Some changes were observed for the correlation coefficients for absolute nutrient intake (in original Table 3) and for food intake (in original Table 5). However, the median correlation coefficients for the absolute nutrient intake and food intake were unchanged. There were some changes for the percentage correctly classified presented in original Table 4, however the median percentage was unchanged.

The identified error does not have consequences for the nutrient density results or for any of the results from the weighed food record. The recalculated and correct values for all the results based on absolute nutrient intake and food intake are presented in Table A and Table B below.

The validity of the SFFQ related to different background parameters are only presented in the text in the original paper (page 759-760). The identified error results in minor changes for the validity according to mothers' education and if the child are in day care or not. Larger changes were observed for the validity according to siblings or not; the correlation coefficients for most micronutrients observed for the group of children with siblings were significant different from those observed among the children without siblings (in the original paper significant differences were found for macronutrients).

Table A Daily intake of energy and nutrients based on the SFFQ and WR (supplements are included) with values expressed as median $\left(P_{25}, P_{75}\right)$, Spearman's $r$ and classification in to quartiles $(n=187)$

\begin{tabular}{|c|c|c|c|c|c|}
\hline \multirow[b]{2}{*}{ Nutrients } & \multicolumn{2}{|c|}{ Absolute intaket } & \multirow{2}{*}{$\frac{\text { Absolute intaket }}{\text { Spearman's } r}$} & \multicolumn{2}{|c|}{ Absolute intaket } \\
\hline & SFFQ & WR & & Correctly classified (\%) & Grossly Misclassified (\%) \\
\hline Energy & $5652(4852,6550)$ & $4161(3713,4535)^{\star \star \star}$ & 0.14 & 33 & 9 \\
\hline Protein & $44.4(38.0,50.8)$ & $32.9(28.4,37.3)^{\star \star \star}$ & $0.29^{\star \star \star}$ & 26 & 7 \\
\hline Total fat & $52.4(42.0,64.0)$ & $36.5(31.6,41.0)^{\star * *}$ & $0.25^{\star \star}$ & 33 & 8 \\
\hline SFA & $21.5(17.7,26.2)$ & $15.8(13.8,18.6)^{\star * *}$ & $0.30^{\star \star \star}$ & 32 & 7 \\
\hline MUFA & $15.9(13.0,19.1)$ & $11.5(9.5,13.1)^{\star \star \star}$ & $0.30^{* \star *}$ & 34 & 7 \\
\hline PUFA & $8.1(5.9,11.5)$ & $5.3(4.4,6.6)^{\star \star \star}$ & $0.36^{\star \star \star}$ & 39 & 6 \\
\hline Total carbo & $172.2(148.0,205.0)$ & $131.6(114.2,146.2)^{\star \star \star}$ & $0.22^{\star *}$ & 35 & 7 \\
\hline Sugar $\ddagger$ & $34.2(22.5,48.0)$ & $32.7(23.5,44.0)$ & $0.44^{* * *}$ & 33 & 4 \\
\hline Fiber & $12.2(9.3,15.3)$ & $7.2(5.4,8.9)^{\star \star \star}$ & $0.34^{\star \star \star}$ & 36 & 5 \\
\hline Vitamin A & $1586(1130,2230)$ & $694(473,1027)^{\star \star *}$ & $0.59^{* \star *}$ & 43 & 3 \\
\hline Vitamin D & $9.1(5.8,13.7)$ & $3.9(1.6,7.3)^{\star \star \star *}$ & $0.48^{\star \star \star}$ & 41 & 4 \\
\hline Vitamin E & $9.3(6.2,12.7)$ & $4.7(2.9,7.2)^{\star \star \star}$ & $0.48^{\star \star *}$ & 36 & 3 \\
\hline Thiamin & $1.12(0.86,1.64)$ & $0.66(0.55,0.91)^{\star \star \star}$ & $0.52^{\star \star \star}$ & 44 & 4 \\
\hline Riboflavin & $1.63(1.24,2.24)$ & $1.06(0.84,1.33)^{\star * *}$ & $0.51^{* \star *}$ & 45 & 3 \\
\hline Vitamin C & $88(62,119)$ & $60(42,79)^{\star \star \star}$ & $0.43^{* * *}$ & 42 & 5 \\
\hline Calcium & $697(559,872)$ & $520(406,649)^{\star \star \star}$ & $0.38^{\star \star \star}$ & 32 & 5 \\
\hline Iron & $6.5(5.2,8.0)$ & $4.5(3.6,5.2)^{\star \star \star}$ & $0.42^{\star \star \star}$ & 35 & 1 \\
\hline
\end{tabular}

SFFQ - semi-quantitative food frequency questionnaire; WR - weighed records; $r-$ Spearman's rank correlation coefficient; $P_{25}=25$ th percentiles, $P_{75}=75$ th percentile; SFA - saturated fatty acids; MUFA - monounsaturated fatty acids; PUFA - polyunsaturated fatty acids.

tAbsolute intake: Energy was measured as $\mathrm{kJ}$; Fats, protein and carbohydrates in g; vitamin A and D in $\mu \mathrm{g}$; The other vitamins and minerals in mg $\ddagger$ Added sugar.

$\mathrm{p}<0.05,{ }^{* \star} \mathrm{p}<0.01,{ }^{\star \star *} \mathrm{p}<0.001$ 
The conclusion should be changed to;

"In summary, the present validation study indicates that the SFFQ used among 2-year-olds in Norway overestimates the median intakes of most nutrients and foods. The capability of the questionnaire to rank children according to nutrient intake was low, but increased when using nutrient density values instead of absolute values. The ability to rank children according to foods was moderate. Furthermore, we found that the validity of the SFFQ did not seem to be influenced by length of mothers' education or whether the child was attending day care or not."

Table B Daily intake of different food items ( $g$ per day) based on the SFFQ and WR with values expressed as median $\left(P_{25}, P_{75}\right)$ and Spearman's $r(n=187)$

\begin{tabular}{|c|c|c|c|}
\hline Food & SFFQ & WR & $r$ \\
\hline Bread & $93(63,120)$ & $57(43,78)^{\star \star \star}$ & $0.52^{\star \star \star}$ \\
\hline Cake & $15(9,23)$ & $20(10,35)^{\star \star \star}$ & $0.39^{\star \star \star}$ \\
\hline Potatoes & $19(10,43)$ & $12(5,22)^{\star \star *}$ & $0.28^{\star \star \star}$ \\
\hline Vegetables & $26(13,45)$ & $16(7,30)^{\star \star \star}$ & $0.46^{\star \star \star}$ \\
\hline Fruit, berries & $167(102,267)$ & $112(66,175)^{\star \star \star}$ & $0.57^{\star \star \star *}$ \\
\hline Fruit juice & $34(0,86)$ & $21(0,69)^{\star \star \star}$ & $0.63^{* * *}$ \\
\hline Meat & $42(29,54)$ & $33(21,48)^{\star \star \star}$ & $0.48^{* \star *}$ \\
\hline Fish & $16(10,24)$ & $11(4,21)^{\star \star \star}$ & $0.44^{* \star *}$ \\
\hline Cheese & $14(10,24)$ & $10(6,16)^{\star \star \star}$ & $0.55^{\star \star \star}$ \\
\hline Yoghurt & $81(36,125)$ & $45(13,87)^{\star \star \star}$ & $0.54^{\star \star \star}$ \\
\hline Milk & $269(156,412)$ & $220(114,314)^{\star * *}$ & $0.54^{\star \star \star}$ \\
\hline Soft drinkst with sugar & $86(21,231)$ & $116(51,204)$ & $0.51^{* \star *}$ \\
\hline Soft drinks without sugar & $0(0,0)$ & $0(0,36)^{*}$ & $0.44^{* * *}$ \\
\hline Chocolate, sweets & $3(1,6)$ & $4(1,8)^{\star}$ & $0.32^{* \star *}$ \\
\hline Cod liver oil & $0(0,4)$ & $0(0,1.4)^{\star * \star}$ & $0.71^{* \star *}$ \\
\hline
\end{tabular}

SFFQ - semi-quantitative food frequency questionnaire; WR - weighed records; $P_{25}=25$ th percentiles, $P_{75}=75$ th percentile; $r$ - Spearman's rank correlation coefficient.

tSoft drinks is carbonated soft drinks and squash.

${ }^{*} p<0.05,{ }^{* *} p<0.01,{ }^{* * *} p<0.001$.

\section{Reference}

Andersen LF, Lande B, Trygg K \& Hay G (2004) Validation of a semi-quantitative food-frequency questionnaire used among 2-year-old Norwegian children. Public Health Nutrition 7(6): 757-64. Published online by Cambridge University Press 2 Jan 2007. 